\title{
Fluctuations in a $p$-spin interaction model
}

\author{
Holger Knöpfel ${ }^{\mathrm{a}}$, Matthias Löwe ${ }^{\mathrm{b}, *}$ \\ ${ }^{a}$ Ruhr-Universität Bochum, Fakultät für Mathematik, NA 3/51, 44780 Bochum, Germany \\ ${ }^{\mathrm{b}}$ Westfälische Wilhelms-Universität Münster, Fachbereich Mathematik, Einsteinstraße 62, 48149 Münster, Germany
}

Received 10 November 2003; received in revised form 23 April 2004; accepted 28 May 2004

Available online 12 October 2004

\begin{abstract}
We investigate the behavior of the free energy of the $p$-spin interaction variant of the SK-model for high temperatures. We admit arbitrary distributions of the interactions given that their distribution is symmetric around the origin and some exponential moment is finite. We show that there is a critical temperature $\tilde{\beta}$ depending on $p$ such that for $\beta<\tilde{\beta}$ the free energy of the $p$-spin interaction model has normally distributed fluctuations.
\end{abstract}

(c) 2004 Elsevier SAS. All rights reserved.

\section{Résumé}

Nous étudions le comportement à haute température de l'énergie libre du verre de spin à interactions multiples d'ordre $p$. Pour des corrélations aléatoires admettant un moment exponentiel, nous démontrons un théorème limite central pour l'énergie libre à haute température.

() 2004 Elsevier SAS. All rights reserved.

Keywords: Spin glasses; Central Limit Theorem

\section{Introduction}

Spin glasses have been in the centre of interest of probability theory and statistical mechanics in the past decade. The reader interested in the various aspects of spin glasses is referred to the excellent book by Mèzard et al. [11] for the physicists' point of view or to the lecture notes by Talagrand [14], his very recent book [15] or Bovier's lecture notes [3] for mathematical aspects of the models.

The following model can be considered as a generalization of the famous Sherrington-Kirkpatrick model (SKmodel for short) of spin glasses with interaction (also called coupling) between each $p$-tuple $(p \geqslant 3)$ of spins. However, the reason for considering this model has not been the desire for generalization but the hope that its low

\footnotetext{
* Corresponding author.

E-mail addresses: holger.knoepfel@rub.de (H. Knöpfel), maloewe@ math.uni-muenster.de (M. Löwe).
} 
temperature behavior is much simpler than that of the SK-model, so that it should be regarded first. Indeed, such an analysis is nowadays largely available, see the article by Talagrand [12] or his book [15, Chapter 6].

To define the $p$-spin interaction model we shall start with a sequence $\left(\sigma_{i}\right)_{i \in \mathbb{N}}$ of independent random variables $\sigma_{i}: \Omega_{\sigma} \rightarrow\{-1,1\}$ with $\mathbb{P}_{\sigma}\left(\sigma_{i}=-1\right)=\mathbb{P}_{\sigma}\left(\sigma_{i}=1\right)=1 / 2$. Introducing the notation $\vec{\imath} \in\left(\begin{array}{l}N \\ p\end{array}\right)$ for $\vec{\imath}=\left(i_{1}, \ldots, i_{p}\right)$

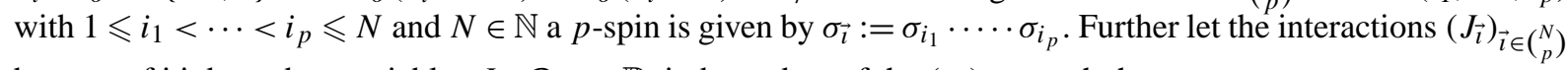
be a set of i.i.d. random variables $J_{i}: \Omega_{J} \rightarrow \mathbb{R}$, independent of the $\left(\sigma_{i}\right)_{i \in \mathbb{N}}$ such that

- The distribution of $J_{\vec{l}}$ is symmetric (with respect to the origin).

- $J_{\vec{i}}$ has exponential moments, i.e. $\mathbb{E}_{J} \exp \left(t J_{\vec{l}}\right)<\infty$ for some $t>0$.

- The variance of $J_{\vec{l}}$ is 1 .

Of course, we have $\mathbb{E}_{J} J_{\vec{l}}=0$ with $\mathbb{E}_{\sigma}$ and $\mathbb{E}_{J}$ denoting the expectation with respect to the probability distributions $\mathbb{P}_{\sigma}$ (of $\sigma$ ) and $\mathbb{P}_{J}$ (of $J$ ) respectively.

Let finally the Hamiltonian $H_{N}: \Omega_{\sigma} \times \Omega_{J} \rightarrow \mathbb{R}$ be given by

$$
H_{N}:=-\sqrt{\frac{p !}{N^{p-1}}} \sum_{\vec{\imath} \in\left(\begin{array}{l}
N \\
p
\end{array}\right)} J_{\vec{\imath}} \sigma_{\vec{\imath}} .
$$

Our main object of interest is the behavior of the free energy of the model.

To introduce it let

$$
Z_{N}:=\mathbb{E}_{\sigma} \exp \left(-\beta H_{N}\right): \Omega_{J} \rightarrow \mathbb{R}_{+}
$$

be the partition function at inverse temperature $\beta>0$. The free energy of the system is defined as

$$
f_{N}:=\frac{1}{N} \log Z_{N}
$$

We will be interested in the thermodynamic limit of the quantities $Z_{N}$ and $f_{N}$ (the limit as $N \rightarrow \infty$ ) which in fact strongly depends on $\beta$. Naively, we could expect $Z_{N}$ to obey a Law of Large Numbers as $N$ tends to infinity, i.e. we would expect that $Z_{N}$ behaves like $\mathbb{E}_{J} Z_{N}$ or rather that

$$
f_{N} \rightarrow f^{a}:=\lim _{N \rightarrow \infty} \frac{1}{N} \log \mathbb{E}_{J} Z_{N}
$$

(in probability as $N \rightarrow \infty$ ). It is not difficult to check that the limit on the right-hand side of (1.1) exists and equals $\frac{\beta^{2}}{2}$ (under the assumptions on the interactions stated above). This limit $f^{a}$ in (1.1) is called the annealed free energy. On the other hand one would expect the random variable $f_{N}$ to behave like its average $\mathbb{E}_{J} f_{N}$, i.e. we would expect that

$$
f_{N} \rightarrow f^{q}:=\lim _{N \rightarrow \infty} \mathbb{E}_{J} \frac{1}{N} \log Z_{N}
$$

(in probability as $N \rightarrow \infty$ ) provided the latter limit exists. The limit $f^{q}$ in (1.2) is called the quenched free energy. But (1.1) and (1.2) are likely to contradict each other because by Jensen's inequality

$$
\mathbb{E}_{J} \frac{1}{N} \log Z_{N} \leqslant \frac{1}{N} \log \mathbb{E}_{J} Z_{N}
$$

Indeed, it turns out that a form of (1.2), namely

$$
\lim _{N \rightarrow \infty} \mathbb{P}_{J}\left(\left|f_{N}-\mathbb{E}_{J} f_{N}\right| \geqslant \varepsilon\right)=0
$$

is true for all temperatures, while the validity of $f^{a}=f^{q}$ and hence that of (1.1) is a genuine high temperature phenomenon. 
The critical temperature $\beta_{c}$ of the $p$-spin SK-model depends on $p$ and is defined as the largest $\beta$ such that (1.1) holds, i.e.

$$
\beta_{c}:=\sup \left\{\beta>0: \lim _{N \rightarrow \infty} f_{N}=f^{a} \text { in probability }\right\} .
$$

An example to be kept in mind is that of the couplings $\left(J_{\vec{l}}\right)_{\vec{l}}$ being i.i.d.and standard Gaussians, i.e. $\mathcal{N}(0,1)$ distributed. This is in fact the only example which has been rigorously investigated so far for $p \geqslant 3$ (see the contributions by Talagrand [12], [15], by Bovier et al. [4] and Kurkova [10]). Note that the definition of the critical temperature agrees with the result for the critical temperature in the usual SK-model, where one can show $\beta_{c}=1$ (see e.g. $[1,5,6,13])$. In contrast to the case $p=2$ one has not yet been able to calculate the critical temperature for $p \geqslant 3$, not even for i.i.d.standard Gaussian couplings. For the latter case Talagrand [12] gives the bounds

$$
\inf _{0<t<1} \varphi(t)\left(1+t^{-p}\right) \leqslant \beta_{c}^{2} \leqslant 2 \log 2 \quad \text { with } \varphi(t)=\frac{1}{2}((1+t) \log (1+t)+(1-t) \log (1-t)) .
$$

Moreover it can be proven that for Gaussian couplings $\beta_{c}^{2} \rightarrow 2 \log 2$ as $p \rightarrow \infty$. This could also have been expected since $\sqrt{2 \log 2}$ is the critical temperature in the Random Energy Model [7,9], where the Hamiltonian is the sum of $2^{N}$ independent Gaussian variables.

It is easy to see that indeed the limit (in probability)

$$
\lim _{N \rightarrow \infty} f_{N}=f^{a}
$$

holds in the entire high temperature regime, that is for all $\beta<\beta_{c}$ (basically because the function $\exp (\beta \cdot)$ becomes "less convex" for small $\beta$ ). This can be considered a Law of Large Numbers for the free energy in the high temperature regime.

In this article we will treat the fluctuations in the sense of a Central Limit Theorem from this Law of Large Numbers. We are inspired by the following result by Bovier et al. [4].

Theorem 1.1 (cf. [4, Theorem 1.2]). Fix $p \geqslant 3$ and assume that the random variables $J_{\vec{\imath}}$ are i.i.d. and $\mathcal{N}(0,1)$ distributed. Then there is a (second) critical temperature $\bar{\beta}_{c}(p)$ such that for all $\beta<\bar{\beta}_{c}(p)$ the following Central Limit Theorem holds:

$$
N^{\frac{p-2}{2}} \log \frac{Z_{N}}{\mathbb{E}_{J} Z_{N}} \stackrel{\mathcal{D}}{\longrightarrow} N \rightarrow \infty \mathcal{N}\left(0, \beta^{4} p ! / 2\right) .
$$

The related question for $p=2$ was answered by Aizenman et al. [1] using combinatorial techniques and by Comets and Neveu [6] via methods from stochastic calculus (which are available for Gaussian couplings $J_{\vec{\imath}}$ only). For $p=2$ an additional centering is necessary, since $\mathbb{E}_{J} \log \frac{Z_{N}}{\mathbb{E}_{J} Z_{N}}$ does not converge to zero in the CLT scaling.

We have been challenged by Theorem 1.1 due to the following reasons. First of all the requirement that the couplings are i.i.d. Gaussians seems to be unnecessarily restrictive for a Central Limit Theorem to hold. Usually one would expect the validity of a Central Limit Theorem to depend on some moment assumptions of the underlying variables, but not on the fact that they are themselves some functions of Gaussian variables. Moreover the proof of Theorem 1.1 seems extraordinarily long (approximately 25 pages) and we were expecting that exploiting the symmetry of the couplings could possibly shorten this proof. Indeed we are presenting an elementary proof of the following result.

Theorem 1.2. Assume that the random variables $J_{\vec{\imath}}$ are i.i.d. and satisfy the conditions stated above. Then there is a temperature $\tilde{\beta}=\tilde{\beta}(p) \leqslant \beta_{c}$ depending on $p$ such that for all $\beta<\tilde{\beta}$ the following CLT holds:

$$
N^{\frac{p-2}{2}} \log \frac{Z_{N}}{\mathbb{E}_{J} Z_{N}} \stackrel{\mathcal{D}}{\longrightarrow} N \rightarrow \infty \mathcal{N}\left(0, \frac{1}{4} \beta^{4} p !\left(\mathbb{E}_{J}\left(J_{\vec{i}}^{4}\right)-1\right)\right) .
$$

Moreover we have $\tilde{\beta}(p) \rightarrow \sqrt{\log 2}$ for $p \rightarrow \infty$. 
Remark 1.3. Recently Ben Arous et al. [2] obtained Laws of Large Numbers and CLTs for a version of the Random Energy Model with arbitrary (independent) random variables for the energy. Their results together with Theorem 1.1 suggest that Theorem 1.2 should be valid for all $\beta$ less than a critical temperature $\beta_{c}(p)$ that depends on the tail behavior of the couplings $J_{i}$ and in the case of Gaussian couplings converges to $\sqrt{2 \log 2}$ as $p$ tends to infinity. At the present stage we do not have a proof for a statement of that kind. However, Theorem 1.2 shows the universality of a CLT for the free energy for temperatures below $\tilde{\beta}(p)$.

From Theorem 1.2 we readily deduce the corresponding Weak Law of Large Numbers (which has only been established for Gaussian interactions) and hence a bound on the critical temperature that is independent of the specific distribution of the interactions.

Corollary 1.4. Under the assumptions of Theorem 1.2 the free energy $f_{N}$ satisfies a Weak Law of Large Numbers for all $\beta<\tilde{\beta}$.

The proof of Theorem 1.2 starts with the useful decomposition of the partition function as in the paper by Aizenman et al. [1] while later on we use the particular structure of the $p$-spin models $(p \geqslant 3)$ that simplifies computations.

\section{Proof}

We start with the simple identity $\mathrm{e}^{x}=\cosh (x) \cdot(1+\tanh (x))$. Using the odd symmetry of tanh and the even symmetry of cosh we obtain $Z_{N}=\bar{Z}_{N} \cdot \widehat{Z}_{N}$ where

$$
\bar{Z}_{N}=\prod_{\vec{\imath} \in\left(\begin{array}{c}
N \\
p
\end{array}\right)} \cosh \left(\beta \sqrt{\frac{p !}{N^{p-1}}} J_{\vec{\imath}}\right) \text { and } \widehat{Z}_{N}=\mathbb{E}_{\sigma} \prod_{\vec{\imath} \in\left(\begin{array}{l}
N \\
p
\end{array}\right)}\left(1+\sigma_{\vec{\imath}} \tanh \left(\beta \sqrt{\frac{p !}{N^{p-1}}} J_{\vec{\imath}}\right)\right) .
$$

Therefore

$$
N^{\frac{p-2}{2}} \log \frac{Z_{N}}{\mathbb{E}_{J} Z_{N}}=N^{\frac{p-2}{2}} \log \frac{\bar{Z}_{N}}{\mathbb{E}_{J} Z_{N}}+N^{\frac{p-2}{2}} \log \widehat{Z}_{N}
$$

Notice, that $\mathbb{E}_{J} Z_{N}=\mathbb{E}_{\sigma} \mathbb{E}_{J} \mathrm{e}^{-\beta H_{N}}=\prod_{\vec{l}} \mathbb{E}_{J} \cosh \left(\beta \sqrt{\frac{p !}{N^{p-1}}} J_{\vec{l}}\right)$ due to the symmetry and independence of the $J_{\vec{\imath}}$ 's. We first aim at showing

$$
N^{\frac{p-2}{2}} \log \frac{\bar{Z}_{N}}{\mathbb{E}_{J} Z_{N}} \stackrel{\mathcal{D}}{\longrightarrow} N \rightarrow \infty \mathcal{N}\left(0, \frac{p ! \beta^{4}}{4}\left(\mathbb{E}_{J} J_{\vec{\imath}}^{4}-1\right)\right) .
$$

Indeed, since

$$
\mathbb{E}_{J} \cosh \left(\beta \sqrt{\frac{p !}{N^{p-1}}} J_{\vec{\imath}}\right)=1+\frac{p ! \beta^{2}}{2 N^{p-1}}+\mathcal{O}\left(N^{2(1-p)}\right)
$$

we obtain

$$
\begin{aligned}
N^{\frac{p-2}{2}} \log \frac{\bar{Z}_{N}}{\mathbb{E} J_{J} Z_{N}} & =N^{\frac{p-2}{2}} \sum_{\vec{\imath} \in\left(\begin{array}{c}
N \\
p
\end{array}\right)}\left(\log \cosh \left(\beta \sqrt{\frac{p !}{N^{p-1}}} J_{\vec{\imath}}\right)-\frac{p ! \beta^{2}}{2 N^{p-1}}\right)+\mathcal{O}\left(N^{1-\frac{p}{2}}\right) \\
& =\sqrt{\frac{p !}{N^{p}}} \sum_{\vec{\imath} \in\left(\begin{array}{c}
N \\
p
\end{array}\right)} \frac{\sqrt{p !} \beta}{2}\left(J_{\vec{\imath}}^{2}-1\right)+N^{\frac{p-2}{2}} \sum_{\vec{\imath} \in\left(\begin{array}{c}
N \\
p
\end{array}\right)} R_{\vec{\imath}}+\mathcal{O}\left(N^{1-\frac{p}{2}}\right)
\end{aligned}
$$


with $R_{\vec{l}}=\log \cosh \left(\beta \sqrt{\frac{p !}{N^{p-1}}} J_{\vec{l}}\right)-\frac{p ! \beta^{2}}{2 N^{p-1}} J_{\vec{l}}^{2}$. Now the first term by the CLT for sequences of independent random variables converges as proposed in (2.2) and for the remaining term we have $L^{1}$-convergence to zero because $\mathrm{e}^{\frac{x^{2}}{2}} \geqslant \cosh (x) \geqslant \mathrm{e}^{\frac{x^{2}}{2}-\frac{x^{4}}{12}}$ and therefore $0 \geqslant R_{\vec{\imath}} \geqslant-\frac{p ! \beta^{4}}{12 N^{2(p-1)}} J_{\vec{i}}^{4}$.

The rest of this paper is devoted to the proof of the following lemma.

Lemma 2.1. For every $\beta<\tilde{\beta}$ (with $\tilde{\beta}$ specified in (2.5) below) we have

$$
N^{\frac{p-2}{2}} \log \widehat{Z}_{N} \stackrel{\mathcal{D}}{\longrightarrow} N \rightarrow \infty 0 .
$$

Proof. For the proof we fix $\varepsilon>0$ and observe that for large $N$ by Chebyshev's inequality

$$
\mathbb{P}_{J}\left(N^{\frac{p-2}{2}}\left|\log \widehat{Z}_{N}\right| \geqslant \varepsilon\right) \leqslant \mathbb{P}_{J}\left(\left|\widehat{Z}_{N}-1\right| \geqslant \frac{\varepsilon}{2} N^{\frac{2-p}{2}}\right) \leqslant \frac{4}{\varepsilon^{2}} N^{p-2} \mathbb{E}_{J}\left(\widehat{Z}_{N}-1\right)^{2} .
$$

Now taking a closer look at $\widehat{Z}_{N}$ by simply expanding the product we obtain

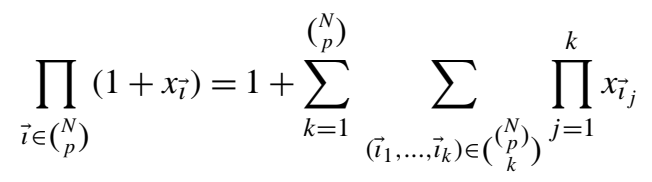

where here $x_{\vec{\imath}}=\sigma_{\vec{\imath}} t_{\vec{\imath}}$ and $t_{\vec{\imath}}=\tanh \left(\beta \sqrt{p ! / N^{p-1}} J_{\vec{\imath}}\right)$. Obviously $\mathbb{E}_{\sigma} \prod_{j=1}^{k} \sigma_{\vec{\imath} j}=0$ whenever we can find an index $i \in\{1, \ldots, N\}$ occurring an odd number of times in the $k \cdot p$-tuple $\left(\vec{\imath}_{1}, \ldots, \vec{\imath}_{k}\right)$. In contrast let $\mathcal{E}_{k}$ denote the set of all $\left(\vec{\imath}_{1}, \ldots, \vec{\imath}_{k}\right)$ having an even number of occurrences for each index that appears then we clearly have $\prod_{j=1}^{k} \sigma_{\vec{\imath}_{j}}=1$ for these $k \cdot p$-tuples and therefore

$$
\widehat{Z}_{N}=1+\sum_{k=1}^{\left(\begin{array}{c}
N \\
p
\end{array}\right)} \sum_{\left(\vec{\imath}_{1}, \ldots, \vec{\imath}_{k}\right) \in \mathcal{E}_{k}} \prod_{j=1}^{k} t_{\vec{l}_{j}}=1+\sum_{\xi \in \mathcal{E}} \tau_{\xi}
$$

with $\mathcal{E}:=\bigcup_{k=1}^{\left(\begin{array}{c}N \\ p\end{array}\right)} \mathcal{E}_{k}$ and the obvious meaning for $\tau_{\xi}$. Now by the properties of the variables $\left(J_{\vec{l}}\right)_{\vec{\imath}}$ we have $\mathbb{E}_{j}\left(\tau_{\xi} \tau_{\zeta}\right)=0$ for $\xi \neq \zeta$ so that $\mathbb{E}_{J}\left(\widehat{Z}_{N}-1\right)^{2}=\sum_{\xi \in \mathcal{E}} \mathbb{E}_{J} \tau_{\xi}^{2}$. Using $\tanh ^{2} x \leqslant x^{2}$ and $\mathbb{E}_{J} J_{\vec{i}}^{2}=1$ we get

$$
0 \leqslant \mathbb{E}_{J}\left(\widehat{Z}_{N}-1\right)^{2}=\sum_{k=1}^{\left(\begin{array}{c}
N \\
p
\end{array}\right)} \sum_{\left(\overrightarrow{\vec{l}}_{1}, \ldots, \vec{\imath}_{k}\right) \in \mathcal{E}_{k}} \prod_{j=1}^{k} \mathbb{E}_{J} t_{\vec{l}_{j}}^{2} \leqslant \sum_{k=1}^{\left(\begin{array}{c}
N \\
p
\end{array}\right)}\left(\frac{\beta^{2} p !}{N^{p-1}}\right)^{k}\left|\mathcal{E}_{k}\right|=\mathbb{E}_{\sigma} \prod_{\vec{\imath} \in\left(\begin{array}{c}
N \\
p
\end{array}\right)}\left(1+\sigma_{\vec{\imath}} \frac{\beta^{2} p !}{N^{p-1}}\right)-1 .
$$

Therefore the proof is complete once we have established the following lemma.

Lemma 2.2. For every $\beta<\tilde{\beta}$ (with $\tilde{\beta}$ specified in (2.5)) we have

$$
\lim _{N \rightarrow \infty} N^{p-2}\left(\mathbb{E}_{\sigma} \prod_{\vec{\imath} \in\left(\begin{array}{c}
N \\
p
\end{array}\right)}\left(1+\sigma_{\vec{\imath}} \frac{\beta^{2} p !}{N^{p-1}}\right)-1\right)=0 .
$$

Remark 2.3. Notice that the event $\left\{\sigma_{i}=1,1 \leqslant i \leqslant N\right\}$ enforces

$$
\mathbb{E}_{\sigma} \prod_{\vec{\imath} \in\left(\begin{array}{c}
N \\
p
\end{array}\right)}\left(1+\sigma_{\vec{\imath}} \frac{\beta^{2} p !}{N^{p-1}}\right) \geqslant 2^{-N}\left(1+\frac{\beta^{2} p !}{N^{p-1}}\right)^{\left(\begin{array}{c}
N \\
p
\end{array}\right)}
$$


and therefore $\tilde{\beta} \leqslant \sqrt{\log 2}$ is unavoidable at this stage of the proof. Of course, rather than being coincidental this points to the heart of the (mathematical) meaning of $\beta$. In fact, using $1+x=\mathrm{e}^{x-\frac{x^{2}}{2}+\mathrm{O}\left(x^{3}\right)}$ we obtain

$$
\prod_{\vec{\imath} \in\left(\begin{array}{l}
N \\
p
\end{array}\right)}\left(1+\sigma_{\vec{\imath}} \frac{\beta^{2} p !}{N^{p-1}}\right)=\exp \left(\frac{\beta^{2} p !}{N^{p-1}} S_{N}-\frac{1}{2}\left(\begin{array}{c}
N \\
p
\end{array}\right) \frac{\beta^{4} p !^{2}}{N^{2(p-1)}}+\mathrm{O}\left(N^{3-2 p}\right)\right)
$$

where $S_{N}=\sum_{\vec{\imath} \in\left(\begin{array}{c}N \\ p\end{array}\right)} \sigma_{\vec{\imath}}$.

The next lemma treats the $L^{1}$-boundedness of (2.4), i.e. the boundedness of

$$
\left\{\mathbb{E}_{\sigma} \exp \left(\frac{\beta^{2} p !}{N^{p-1}} S_{N}\right)\right\}_{N \in \mathbb{N}},
$$

which is certainly necessary for Lemma 2.2 to hold and substantially depends on $\beta$.

Lemma 2.4. For every $\beta<\tilde{\beta}$ (with $\beta$ specified in (2.5)) the sequence

$$
\left\{\mathbb{E}_{\sigma} \exp \left(\frac{\beta^{2} p !}{N^{p-1}} S_{N}\right)\right\}_{N \in \mathbb{N}}
$$

is bounded.

Proof. It suffices to prove the boundedness of

$$
\left\{\mathbb{E}_{\sigma} \exp \left(\frac{\beta^{2}}{N^{p-1}} T_{N}^{p}\right)\right\}_{N \in \mathbb{N}}
$$

with $T_{N}=\sum_{i=1}^{N} \sigma_{i}$. Now fix $0<\beta<\tilde{\beta}$ and observe that

$$
\mathbb{E}_{\sigma} \exp \left(\beta^{2} N\left(\frac{T_{N}}{N}\right)^{p}\right) \leqslant 1+\int_{1}^{\mathrm{e}^{\beta^{2} N}} \mathbb{P}_{\sigma}\left(\left(\frac{T_{N}}{N}\right)^{p} \geqslant \frac{\log y}{\beta^{2} N}\right) \mathrm{d} y .
$$

Clearly $\mathbb{P}_{\sigma}\left(\left(\frac{T_{N}}{N}\right)^{p} \geqslant \frac{\log y}{\beta^{2} N}\right) \leqslant 2 \exp \left(-N \cdot \mathcal{I}\left(\sqrt[p]{\frac{\log y}{\beta^{2} N}}\right)\right)$ where $\mathcal{I}(z)=\int_{0}^{z} \operatorname{artanh} t \mathrm{~d} t$ is the ordinary entropy of a single spin. Therefore inferring

$$
\mathcal{I}(\sqrt[p]{z})=\sum_{n=1}^{\infty} \frac{z^{2 n / p}}{2 n(2 n-1)} \geqslant z \sum_{n=1}^{\lfloor p / 2\rfloor} \frac{1}{2 n(2 n-1)}=: z \cdot \tilde{\beta}^{2}
$$

for $0 \leqslant z \leqslant 1$ we obtain

$$
\int_{1}^{\mathrm{e}^{\beta^{2} N}} \mathrm{e}^{-N \cdot \mathcal{I}\left(\sqrt[p]{\frac{\log y}{\beta^{2} N}}\right)} \mathrm{d} y \leqslant \int_{1}^{\mathrm{e}^{\beta^{2} N}} y^{-\frac{\tilde{\beta}^{2}}{\beta^{2}}} \mathrm{~d} y \leqslant\left(\left(\frac{\tilde{\beta}}{\beta}\right)^{2}-1\right)^{-1}
$$

which proves the lemma.

Remark 2.5. Observe that $\lim _{p \rightarrow \infty} \tilde{\beta}(p)^{2}=\mathcal{I}(1)=\log 2$.

With this uniform integrability statement at hand we now prove Lemma 2.2. 
Proof of Lemma 2.2. In view of (2.4) we are left to prove that for $\beta<\tilde{\beta}$

$$
\lim _{N \rightarrow \infty} N^{p-2}\left(\mathbb{E}_{\sigma} \mathrm{e}^{M_{N}}-\mathrm{e}^{\frac{1}{2} \mathbb{V}_{\sigma} M_{N}}\right)=0
$$

Here we have set $M_{N}:=\frac{\beta^{2} p !}{N^{p-1}} S_{N}$ and have used the pairwise independence of the $\left(\sigma_{\vec{\imath}}\right)_{\vec{\imath}}$ to obtain the variance $\mathbb{V}_{\sigma} S_{N}=\left(\begin{array}{l}N \\ p\end{array}\right)$ of $S_{N}$. Recall that we already know the limit in (2.6) to be non-negative due to (2.3). Now making use of the inequality

$$
\mathrm{e}^{x} \leqslant 1+x+\frac{x^{2}}{2}+\frac{x^{3}}{3 !} \mathrm{e}^{v} \cdot \mathbf{1}_{\{0 \leqslant x<v\}}+\mathrm{e}^{x} \cdot \mathbf{1}_{\{x \geqslant v\}}, \quad x \in \mathbb{R}, v>0
$$

with $x=M_{N}$ and $v=N^{\frac{2-p}{3}}$ Eq. (2.6) will clearly follow if we can establish

$$
\lim _{N \rightarrow \infty} \mathbb{E}_{\sigma}\left(\left(\frac{M_{N}}{v}\right)^{3} \cdot \mathbf{1}_{\left\{0 \leqslant M_{N}<v\right\}}\right)=0
$$

and

$$
\lim _{N \rightarrow \infty} N^{p-2} \mathbb{E}_{\sigma}\left(\mathrm{e}^{M_{N}} \cdot \mathbf{1}_{\left\{M_{N} \geqslant v\right\}}\right)=0
$$

for $\beta<\tilde{\beta}$. But since

$$
\mathbb{V}_{\sigma}\left(\frac{M_{N}}{v}\right)=\beta^{4}(p !)^{2} v^{-2} N^{2(1-p)}\left(\begin{array}{c}
N \\
p
\end{array}\right)=\mathcal{O}\left(N^{\frac{2-p}{3}}\right)
$$

we have $\frac{M_{N}}{v} \stackrel{\mathcal{D}}{\longrightarrow} 0$ which implies (2.7). In order to prove (2.8) we fix $\beta<\tilde{\beta}$ and $\delta>0$ with $\beta^{2}(1+\delta)<\tilde{\beta}^{2}$. Now applying Hölder's inequality with conjugate exponents $r$ and $r^{\prime}:=1+\delta$ to (2.8) we see that $\mathbb{E}_{\sigma} \mathrm{e}^{r^{\prime} M_{N}}$ is bounded due to Lemma 2.4. So finally (2.8) is established by the following lemma.

Lemma 2.6. For each $a>0$ the probability

$$
\mathbb{P}_{\sigma}\left(p ! S_{N} \geqslant N^{\frac{p}{2}+a}\right)
$$

decays exponentially fast when $N$ tends to infinity. In particular, for fixed $r>1$

$$
\lim _{N \rightarrow \infty} N^{p-2} \mathbb{P}_{\sigma}^{1 / r}\left(M_{N} \geqslant v\right)=0
$$

Remark 2.7. Of course, a result like Lemma 2.6 can in principle be derived from Moderate Deviation Theorems as proved in [8] (see also references therein). However, we felt that at this stage it would be nice to keep this paper self-contained, in particular as the proof of Lemma 2.6 is not very long.

Proof. Writing $T_{n}=\sum_{i=1}^{N} \sigma_{i}$ and $G_{p, N}=p ! S_{N}$ we see that

$$
\begin{aligned}
T_{N} \cdot G_{p, N} & =\left(\sum_{i=1}^{N} \sigma_{i}\right) \cdot\left(\sum_{i_{1} \neq \cdots \neq i_{p}} \sigma_{i_{1}} \cdots \cdots \sigma_{i_{p}}\right) \\
& =\sum_{i \neq i_{1} \neq \cdots \neq i_{p}} \sigma_{i} \cdot \sigma_{i_{1}} \cdots \sigma_{i_{p}}+p \sum_{i_{1} \neq \cdots \neq i_{p}} \sigma_{i_{1}} \cdots \sigma_{i_{p-1}} \\
& =G_{p+1, N}+p(N-p+1) G_{p-1, N}
\end{aligned}
$$

or equivalently

$$
G_{p, N}=T_{N} \cdot G_{p-1, N}-(p-1)(N-p+2) G_{p-2, N}
$$


with $G_{1, N}=T_{N}$ and $G_{0, N}=1$. While solving this recursion relation may be demanding we just need simple mathematical induction to verify the ansatz

$$
G_{p, N}=T_{N}^{p}+\sum_{k=1}^{\lfloor p / 2\rfloor} Q_{k}(N) T_{N}^{p-2 k}
$$

where $Q_{k}(N)$ is a polynomial in $N$ of degree $k$ with coefficients depending on $p$ only. Now setting $Q_{0}(N) \equiv 1$ and using (2.10) we get

$$
\begin{aligned}
& \mathbb{P}_{\sigma}\left(p ! S_{N} \geqslant N^{\frac{p}{2}+a}\right) \\
& \leqslant \sum_{k=0}^{\lfloor p / 2\rfloor} \mathbb{P}_{\sigma}\left(Q_{k}(N) \cdot T_{N}^{p-2 k} \geqslant \frac{1}{1+\left\lfloor\frac{p}{2}\right\rfloor} \cdot N^{\frac{p}{2}+a}\right) \\
& \leqslant p \cdot \max _{0 \leqslant k \leqslant\left\lfloor\frac{p}{2}\right\rfloor} \mathbb{P}_{\sigma}\left(\left|\frac{T_{N}}{\sqrt{N}}\right|^{p-2 k} \geqslant c_{p} \cdot N^{a}\right) \\
& \quad=p \cdot \mathbb{P}_{\sigma}\left(\left|\frac{T_{n}}{\sqrt{N}}\right| \geqslant C_{p} \cdot N^{a / p}\right)
\end{aligned}
$$

where $C_{p}>0$ may change from line to line. The symmetry of $T_{N}$ provides

$$
\mathbb{P}_{\sigma}\left(\left|T_{N}\right| \geqslant C_{p} \cdot N^{\frac{1}{2}+\frac{a}{p}}\right) \leqslant 2 \mathrm{e}^{-N \cdot \mathcal{I}\left(C_{p} N^{\frac{a}{p}-\frac{1}{2}}\right)}
$$

where $\mathcal{I}(z)=\int_{0}^{z} \operatorname{artanh} t \mathrm{~d} t$ whenever $0 \leqslant z \leqslant 1$ and $\mathcal{I}(z)=+\infty$ for $z>1$. Observing $\mathcal{I}(z) \geqslant \frac{z^{2}}{2}$ we finally obtain a decay of at least $\mathrm{e}^{-C_{p} N^{2 a / p}}$ which finishes the proof.

\section{Acknowledgements}

We thank two anonymous referees for an extremely careful reading of an earlier draft of this paper and for bringing Ref. [10] to our attention.

\section{References}

[1] M. Aizenman, J.L. Lebowitz, D. Ruelle, Some rigorous results on the Sherrington-Kirkpatrick spin glass model, Comm. Math. Phys. 112 (1987) 3-20.

[2] G. Ben Arous, L. Bogachev, S. Molchanov, Limit laws for sums of random exponentials, preprint, 2003.

[3] A. Bovier, Statistical Mechanics of Disordered Systems, MaPhySto Lecture Notes, vol. 10, Aarhus, 2001.

[4] A. Bovier, I. Kurkova, M. Löwe, Fluctuations of the free energy in the REM and the p-spin SK-model, Ann. Prob. 30 (2002) $605-651$.

[5] F. Comets, A spherical bound for the Sherrington-Kirkpatrick model, Hommage à P.A. Meyer et J. Neveu, Astérisque 236 (1996) $103-108$.

[6] F. Comets, J. Neveu, The Sherrington-Kirkpatrick model of spin glasses and stochastic calculus: the high temperature case, Comm. Math. Phys. 166 (1995) 549-564.

[7] B. Derrida, Random energy model: limit of a family of disordered models, Phys. Rev. Lett. 45 (1980) 79-82.

[8] P. Eichelsbacher, U. Schmock, Rank-dependent moderate deviations of $U$-empirical measures in strong topologies, Probab. Theory Related Fields 126 (2003) 61-90.

[9] A. Galvez, S. Martinez, P. Picco, Fluctuations in Derrida's random energy and generalized random energy models, J. Stat. Phys. 54 (1989) 515-529.

[10] I. Kurkova, Fluctuations of the free energy and overlaps in the high temperature $p$-spin SK and Hopfield models, Preprint, 2003.

[11] M. Mèzard, G. Parisi, M. Virasoro, Spin Glass Theory and Beyond, World Scientific Lecture Notes in Physics, vol. 9, World Scientific, Teaneck, NJ, 1987. 
[12] M. Talagrand, Rigorous low temperature results for mean field $p$-spin interaction models, Probab. Theory Related Fields 117 (2000) 303-360.

[13] M. Talagrand, The Sherrington-Kirkpatrick model: a challenge for mathematicians, Probab. Theory Related Fields 110 (1998) $109-176$.

[14] M. Talagrand, Verres de Spin et optimisation combinatoire, Séminaire Bourbaki, Astérisque 266 (2000) $287-317$.

[15] M. Talagrand, Spin Glasses: A Challenge for Mathematicians, Springer, Berlin, 2003. 